\title{
Desempenho produtivo e custos com alimentação de novilhos bubalinos alimentados com dietas com diferentes fontes de lipídeos
}

\author{
Ronaldo Lopes Oliveira' ${ }^{1}$, Diêni Maria Pereira Assunção ${ }^{2}$, Marco Aurélio Alves de Freitas Barbosa ${ }^{3}$, \\ Márcio Machado Ladeira ${ }^{4}$, Manoel Messias Pereira da Silva ${ }^{3}$, Renata Lopes de Oliveira ${ }^{2}$

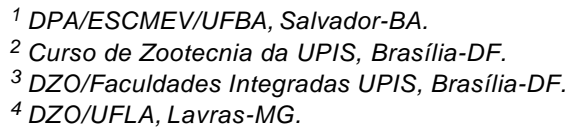

RESUMO - O objetivo neste trabalho foi avaliar o ganho de peso, a conversão alimentar e os custos com alimentação de novilhos bubalinos em sistema de confinamento. Utilizaram-se 13 bubalinos Murrah castrados (peso vivo médio inicial de $297 \pm 9 \mathrm{~kg}$ ) alimentados com três diferentes dietas (tratamentos): sem lipídeo adicional; óleo de soja; e grão de soja integral. A duração do experimento foi de 84 dias, divididos em três períodos de coleta, cada um com 28 dias. Nos seis últimos dias de cada período, foram coletadas amostras de alimentos, sobras e fezes para determinação do consumo. As dietas foram fornecidas duas vezes ao dia (às 8 e 16h), na forma de ração total, em quantidade ajustada para ocorrer 10 a $20 \%$ de sobras. Os animais foram pesados no início do experimento e ao fim de cada período experimental, após jejum de 16 horas. Os ganhos médios diários foram de 990, 1.090 e $1.280 \mathrm{~g} /$ dia para os animais alimentados com as dietas sem lipídeo adicional, com grão de soja e com óleo de soja, respectivamente. As conversões alimentares foram melhores nos animais alimentados com a dieta contendo óleo de soja. O custo da dieta com grão de soja foi maior que o das demais. O melhor desempenho e a melhor resposta econômica foram obtidos quando fornecida a dieta com óleo de soja, o que torna viável sua utilização no confinamento de bubalinos.

Palavras-chave: búfalos, confinamento, grão de soja, óleo de soja

\section{Performance and feeding costs in water buffalo steers fed diets with different fat sources}

\begin{abstract}
The objective of this trial was to evaluate the effect of different dietary fat sources on weight gain, feed conversion, and feeding costs in feedlot water buffalo steers. Thirteen castrated Murrah steers averaging $297 \pm 9 \mathrm{~kg}$ of body weigh were assigned to one of the following three treatments: no added fat (control), soybean oil or whole soybean in a completely randomized design. The experiment lasted 84 days with three experimental periods of 28 days each. Samples of feeds, orts and feces were collected in the last six days of each period for determination of intake. Diets were fed twice a day ( 8 a.m. and 4 p.m.) as total mixed ration and the amount of feed offered was adjusted daily to yield refusals equal to approximately 10 to $20 \%$ of intake. Body weight was measured at the beginning and at the end of each experimental period after 16 hours of fasting. The average daily weight gain was 990, 1,090 and 1,280 g/day for animals fed the control, whole soybean, and soybean oil diets, respectively. Feed conversion was greatest in the diet containing soybean oil while that containing whole soybean resulted in the highest costs. The soybean oil diet increased performance and reduced costs; therefore, it can be used to improve meat production in feedlot water buffalo.
\end{abstract}

Key Words: feedlot, soybean oil, water buffalo, whole soybean

\section{Introdução}

OBrasil tem apresentado números recordes na produção de soja, o que aumenta a disponibilidade de produtos provenientes desta oleaginosa, como farelos e óleo, e favorece a expansão de sistemas de criação intensiva. Outro fator que favorece esta tendência é a sazonalidade da produção de forrageiras, que diminui a oferta de alimentos volumosos na época seca do ano.
A bubalinocultura também tem expandido no País, configurando-se como alternativa para a produção de carne, visto que, a população nacional desses animais em 2003 foi de 1.200 .000 animais (Anualpec, 2004). A produção mundial de carne bubalina foi de 3,17 milhões de toneladas, destacando-se a Índia, o Paquistão e a China como principais produtores no Oriente. No Ocidente, a tendência é que o Brasil se torne o maior produtor de carne bubalina e, 
considerando sua extensão territorial e as condições favoráveis de clima e solo, essa produção pode ser a mais expressiva, a médio e longo prazos, em termos qualiquantitativos. Contudo, a produção e comercialização da carne de búfalos, em boa parte do país, não têm padrão de identificação de suas características, de qualidade ou de valor. Uma vez que não existe diferenciação da carne quanto ao rendimento de cortes comerciais e de carcaça e à qualidade, não há estímulo para modernização do setor produtivo para o fornecimento de um produto mais adequado, que atende às preferências do consumidor (Jorge, 2004).

Entre as vantagens do sistema de terminação em confinamento em relação ao extensivo ou a pasto, destacam-se o melhor controle da alimentação, a redução da idade de abate, a maior produtividade por área, o retorno do capital investido em menor prazo e a produção de carne e carcaças de melhor qualidade. O confinamento contribui para o aumento do usofruto na propriedade, acelera o fluxo produtivo de carne e o giro de capital e, ainda, contribui para a melhoria qualitativa da carne produzida, como ocorre em outros países no mundo (Ramos, 2003). No sistema de confinamento, alguns produtores têm obtido preço até $25 \%$ superior, atribuído à melhor qualidade da carne. Por outro lado, em geral, verifica-se um deságio de aproximadamente $5 \%$ no preço da carne, atribuído ao menor rendimento da carcaça e ao menor aproveitamento do couro, que exige adaptações na tecnologia utilizada pelos frigoríficos e curtumes para seu aproveitamento adequado (Gagleazzil et al., 2003).

Velloso et al. (1994) observaram que bubalinos em regime de confinamento apresentaram desempenho superior ao de zebuínos nelores ( 1,027 vs $0,808 \mathrm{~kg} / \mathrm{dia}$, respectivamente). Ao trabalharem com diversos grupos genéticos, bovinos nelore, mestiços e bubalinos, Jorge et al. (1997) relataram que, quando abatidos em diferentes estádios de maturidade fisiológica, sob idênticas condições de manejo, os búfalos apresentaram potencial para ganho de peso e de carcaça, além de conversão alimentar, semelhante ao de bovinos.

O fornecimento de fontes lipídicas na dieta de ruminantes tem sido adotado no intuito de oferecer alta densidade energética, o que proporciona maiores taxas de ganho de peso e melhor acabamento de carcaça. Contudo, tem-se observado que concentrações lipídicas na dieta superiores a $7 \%$ da MS total alteram o ambiente ruminal e provocam decréscimos na ingestão de MS e na digestibilidade de carboidratos fibrosos, o que diminui o desempenho dos animais (Palmquist, 1994; Jenkins \& McGuire, 2006).

Quando ruminantes recebem fontes de gordura na dieta, os microrganismos do rúmen promovem a hidrólise dos triglicerídeos e a biohidrogenação dos ácidos graxos livres. Os lipídios, quando em excesso na dieta, podem afetar a digestão ruminal e o maior efeito é a redução da digestibilidade da fibra, da produção de metano e da relação acetato:propionato, principalmente se os níveis de ácidos graxos insaturados forem elevados (Hobson \& Stewart, 1997; Doreau \& Ferlay, 1995). Esses efeitos podem ser decorrentes das reduções no crescimento de bactérias (especialmente as celulolíticas/gram positivas) e de protozoários (Tamminga \& Doreau, 1991) e do recobrimento físico da fibra com lipídeos (Hobson \& Stewart, 1997; Jenkins \& McGuire, 2006).

A terminação em confinamento é considerada uma atividade de risco e de baixa lucratividade por unidade de peso ganho, pois é influenciada por fatores externos (taxa cambial, variação internacional de preços, mercado futuro, exportação de carne, consumo interno, entre outros) que nem sempre podem ser controlados pelo produtor. Para quaisquer providências na intensificação da produção, é necessário o conhecimento prévio dos custos envolvidos na atividade e a busca de soluções para diminuí-los sem afetar a produtividade dos animais (Faturi et al., 2003).

Segundo Pacheco et al. (2006), um dos motivos da redução na lucratividade neste segmento consiste no fato de o preço de venda dos animais não ter acompanhado os custos de produção. Nessas situações, a terminação de bovinos de corte deve ser planejada criteriosamente para evitar frustrações econômicas ao produtor.

Este estudo foi realizado com o objetivo de avaliar o ganho de peso, a conversão alimentar e os custos com alimentação de bubalinos Murrah terminados em confinamento com diferentes fontes de lipídeos na dieta.

\section{Material e Métodos}

O experimento foi conduzido na Fazenda Lagoa Bonita - Campus Rural das Faculdades UPIS em Planaltina-DF, no período de setembro a dezembro de 2003. Foram utilizados 13 novilhos bubalinos Murrah castrados com 15 meses de idade e peso vivo inicial de $297 \pm 9 \mathrm{~kg}$. Antes do experimento, os animais foram pesados, vermifugados e distribuídos aleatoriamente em três tratamentos.

As dietas continham silagem de milho como volumoso e milho grão moído, farelo de soja, grão de soja integral ou óleo de soja como ingredientes dos concentrados. Durante todo o período experimental, foram coletadas amostras dos alimentos para análises da composição bromatológica (Tabela 1).

Foram testadas três dietas: sem lipídeo adicional; grão de soja integral; e óleo de soja. A proporção dos ingredientes 
nas dietas e seus valores nutricionais são descritos nas Tabelas 2 e 3, respectivamente.

A duração do experimento foi de 84 dias, divididos em três períodos de coleta, cada um com 28 dias. Nos seis últimos dias de cada período, foram coletadas amostras de alimentos, sobras e fezes. Os animais foram pesados no início do experimento e ao fim de cada período experimental, depois de jejum de 16 horas.

Os animais foram confinados individualmente em baias de madeira, com $24 \mathrm{~m}^{2}$ em piso batido $\left(12 \mathrm{~m}^{2}\right.$ cobertos de telha em fibrocimento). A alimentação foi fornecida em cochos de PVC (3 m lineares/animal) e a água (à vontade), em bebedouro localizado na área descoberta das instalações. A dieta foi fornecida duas vezes ao dia, às 8 e $16 \mathrm{~h}$, na forma de ração total misturada e ajustada para manter as sobras em torno de 10 a $20 \%$ do oferecido. No período da manhã, durante os últimos seis dias de cada período

Tabela 1 - Composição bromatológica dos ingredientes das dietas $(\%)^{1}$

Table 1 - Chemical composition of dietary ingredients

\begin{tabular}{lcrcc}
\hline $\begin{array}{l}\text { Nutriente } \\
\text { Nutrient }\end{array}$ & $\begin{array}{c}\text { Silagem de } \\
\text { milho } \\
\text { Corn } \\
\text { silage }\end{array}$ & $\begin{array}{c}\text { Milho } \\
\text { grão } \\
\text { Corn } \\
\text { grain }\end{array}$ & $\begin{array}{c}\text { Farelo de } \\
\text { soja } \\
\text { Soybean } \\
\text { meal }\end{array}$ & $\begin{array}{c}\text { Grão de soja } \\
\text { integral } \\
\text { Whole } \\
\text { soybean }\end{array}$ \\
\hline MS (DM) (\%) & 32,74 & - & - & - \\
PB (CP) (\%) & 12,60 & 8,32 & 42,80 & 35,77 \\
FDN (NDF) (\%) & 52,63 & 13,55 & 21,12 & 25,56 \\
FDA (ADF) (\%) & 34,34 & 4,03 & 11,90 & 19,72 \\
EE $(\%)$ & 2,96 & 4,74 & 1,50 & 19,55 \\
CNF (NFC) $(\%)$ & 24,01 & 71,82 & 31,17 & 14,55 \\
LIG (\%) & 7,04 & 0,97 & 2,69 & 6,93 \\
\hline
\end{tabular}

${ }^{1}$ Análises realizadas no Laboratório de Análise de Alimentos da UPIS.

${ }_{1}^{1}$ Analysis were done at the UPIS Feed Analysis Laboratory.

Tabela 2 - Proporção dos ingredientes nas dietas (\%MS) Table 2 - Ingredient composition of the experimental diets (\% of DM)

\begin{tabular}{lccc}
\hline $\begin{array}{l}\text { Ingrediente } \\
\text { Ingredient }\end{array}$ & \multicolumn{3}{c}{$\begin{array}{c}\text { Tratamento } \\
\text { Treatment }\end{array}$} \\
\cline { 2 - 4 } & $\begin{array}{c}\text { Sem lipídeo } \\
\text { adicional } \\
\text { No added } \\
\text { fat }\end{array}$ & $\begin{array}{c}\text { Grão de soja } \\
\text { integral } \\
\text { Whole } \\
\text { soybean }\end{array}$ & $\begin{array}{c}\text { Óleo de } \\
\text { soja } \\
\text { Soybean } \\
\text { oil }\end{array}$ \\
\hline $\begin{array}{l}\text { Silagem de milho } \\
\text { Corn silage } \\
\begin{array}{l}\text { Milho grão } \\
\text { Corn grain }\end{array}\end{array}$ & 60,00 & 60,00 & 60,00 \\
$\begin{array}{l}\text { Farelo de soja } \\
\text { Soybean meal } \\
\text { Grão de soja integral }\end{array}$ & 35,17 & 26,41 & 32,09 \\
$\begin{array}{l}\text { Whole soybean } \\
\text { Óleo de soja } \\
\text { Soybean oil }\end{array}$ & 3,71 & - & 4,28 \\
Uréia & - & 13,59 & - \\
Urea & 1,12 & - & 2,50 \\
\hline
\end{tabular}

Tabela 3 - Composição bromatológica (\%MS) das dietas ${ }^{1}$ Table 3 - Chemical composition of the experimental diets (\% of DM)

\begin{tabular}{lccc}
\hline $\begin{array}{l}\text { Nutriente } \\
\text { Nutrient }\end{array}$ & \multicolumn{3}{c}{$\begin{array}{c}\text { Tratamento } \\
\text { Treatment }\end{array}$} \\
\cline { 2 - 4 } & $\begin{array}{c}\text { Sem lipídeo } \\
\text { adicional } \\
\text { No added } \\
\text { fat }\end{array}$ & $\begin{array}{c}\text { Grão de soja } \\
\text { integral } \\
\text { Whole } \\
\text { soybean }\end{array}$ & $\begin{array}{c}\text { Óleo de } \\
\text { soja } \\
\text { Soybean } \\
\text { oil }\end{array}$ \\
\hline PB (CP) & 11,93 & 11,58 & 11,93 \\
FDN (NDF) & 38,54 & 41,01 & 38,20 \\
FDA (ADF) & 23,55 & 27,20 & 23,44 \\
EE (EE) & 3,92 & 5,54 & 6,21 \\
CNF (NFC) & 42,31 & 37,31 & 40,47 \\
Cinzas (Ash) & 3,31 & 4,57 & 3,19 \\
\hline
\end{tabular}

1 Análises realizadas no Laboratório de Análise de Alimentos da UPIS.

${ }^{1}$ Analysis were done at the UPIS Feed Analysis Laboratory.

experimental, foram retiradas e pesadas as sobras para determinação do consumo e cálculo da conversão alimentar.

No Laboratório de Análises de Alimentos do Departamento de Zootecnia da UPIS, foram feitas as análises dos teores de MS, MO, PB e EE, de acordo com a AOAC (1990). As concentrações de fibra em detergente neutro corrigida para cinzas e proteína (FDNcp) e de FDA foram avaliadas segundo Goering \& Van Soest (1970) e o FDNcp dos concentrados, segundo procedimento descrito por Van Soest et al. (1991).

A determinação dos $\mathrm{CNF}$ foi realizada segundo o $\mathrm{NRC}$ (2001), por meio da equação: $\mathrm{CNF}=[100-(\% \mathrm{~PB}+\% \mathrm{FDNcp}$ $+\% \mathrm{EE}+\%$ Cinzas) $]$.

Os custos com alimentação foram calculados considerando os preços de cada ingrediente praticados no mercado de Brasília-DF no segundo semestre de 2003. A lucratividade com a venda da carne foi calculada descontando-se o custo com alimentação neste período do preço pago pelas arrobas ganhas durante os 84 dias de confinamento. Também foi calculada a lucratividade com aluguel da pastagem liberada, considerando o tempo necessário para obter, em pastagem, o ganho de peso obtido em confinamento. Para esta simulação, pressupôs-se que o ganho de peso dos animais a pasto seria em média $450 \mathrm{~g} / \mathrm{cabeça/dia.} \mathrm{A} \mathrm{lucratividade} \mathrm{total}$ correspondeu à soma da lucratividade com a venda da carne mais a lucratividade com o aluguel da pastagem liberada.

Utilizou-se o delineamento experimental inteiramente ao acaso, com repetições no tempo (período) utilizando-se o peso inicial a cada período como covariável. Os dados foram avaliados por meio de análise de variância e as médias pelo teste Tukey a $5 \%$ de probabilidade. $\mathrm{Na}$ análise dos custos com alimentação, não foram utilizadas comparações estatísticas, pois se referiam ao grupo total de animais. 


\section{Resultados e Discussão}

Os valores de ganho de peso médio diário dos animais são descritos na Figura 1.

Os animais alimentados com a dieta contendo óleo de soja apresentaram ganho médio diário de $1.280 \mathrm{~g} / \mathrm{dia}$, superior ao daqueles que consumiram grão de soja $(1.090 \mathrm{~g} / \mathrm{dia})$ e daqueles que receberam a dieta sem lipídeo adicional (990 g/dia), não sendo observada diferença estatística significativa entre esses dois últimos tratamentos. Provavelmente, o maior aporte energético promovido pela dieta com óleo de soja ocasionou os maiores ganhos (Figura 1). Franzolin et al. (2001) estudaram o desempenho de bubalinos alimentados em confinamento com dietas formuladas com três níveis de energia (baixo, médio e alto) e verificaram ganho de 774, 943 e $1.072 \mathrm{~g} / \mathrm{dia}$, respectivamente. Esses resultados comprovam que bubalinos, ao receberem em suas dietas níveis crescentes de energia, tiveram melhores ganhos de peso diário. Entretanto, os valores encontrados nesta pesquisa para a dieta com óleo de soja foram superiores aos observados por esses autores, confirmando o potencial de bubalinos em ganhar peso em sistemas de confinamento com o uso deste co-produto como fonte energética.

Apesar do maior teor de EE, a dieta com grão de soja não diferiu daquela sem lipídeo adicional quanto ao nível de energia e, conseqüentemente, quanto ao ganho de peso, o que pode estar relacionado ao alto teor de FDA do grão de soja. Outra explicação pode ser a disponibilidade mais lenta do óleo presente no grão de soja (Chouinard, 2001).

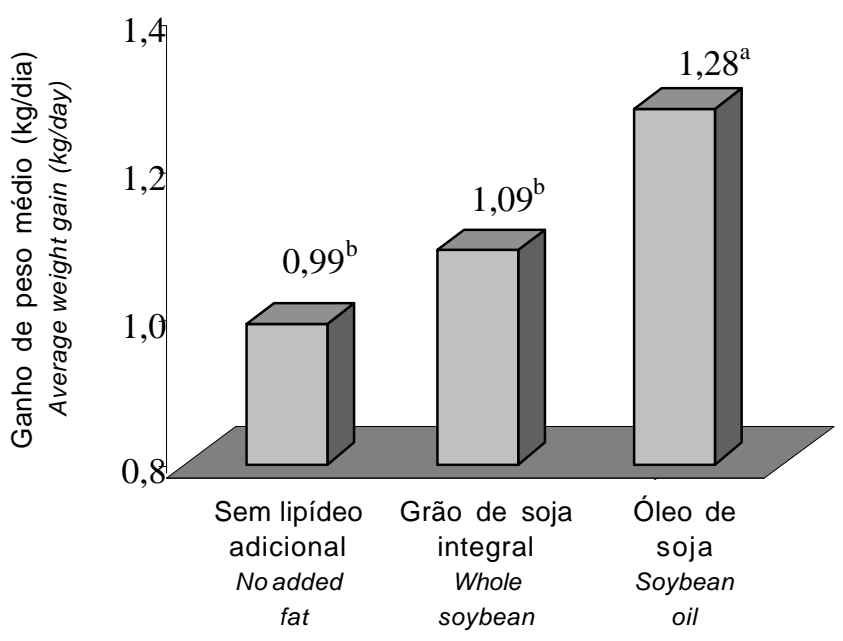

Figura 1 - Ganho de peso médio diário de bubalinos alimentados com diferentes fontes de lipídeos na dieta.

Figure 1 - Daily weight gain of water buffaloes fed diets with different fat sources.

a, b Médias seguidas de mesma letra não diferem estatisticamente pelo teste de Tukey $(P>0,05)$.

$a, b$ Means followed by the same letter do not differ $(P>0.05)$ statistically by Tukey test.
Tabela 4 - Conversão alimentar de nutrientes ( $\mathrm{kg} / \mathrm{kg}$ de ganho) Table 4 - Feed conversion of different nutrients ( $\mathrm{kg} / \mathrm{kg}$ gained)

\begin{tabular}{lccrr}
\hline $\begin{array}{l}\text { Nutriente } \\
\text { Nutrient }\end{array}$ & \multicolumn{3}{c}{$\begin{array}{c}\text { Tratamento } \\
\text { Treatment }\end{array}$} & CV (\%) \\
\cline { 2 - 4 } & $\begin{array}{c}\text { Sem lipídeo } \\
\text { adicional } \\
\text { No added }\end{array}$ & $\begin{array}{c}\text { Grão de soja } \\
\text { integral } \\
\text { fat }\end{array}$ & $\begin{array}{c}\text { Óleo de } \\
\text { Soja }\end{array}$ & Soybean \\
& soybean & oil & \\
\hline MS (DM) & $10,45^{\mathrm{a}}$ & $8,33^{\mathrm{ab}}$ & $7,78^{\mathrm{b}}$ & 12,65 \\
PB (CP) & $1,71^{\mathrm{a}}$ & $1,37^{\mathrm{b}}$ & $1,24^{\mathrm{b}}$ & 9,94 \\
FDN (NDF) & $3,58^{\mathrm{a}}$ & $2,89^{\mathrm{b}}$ & $2,88^{\mathrm{b}}$ & 17,18 \\
NDT (TDN) & $7,52^{\mathrm{a}}$ & $6,12^{\mathrm{b}}$ & $5,44^{\mathrm{b}}$ & 10,77 \\
\hline
\end{tabular}

a, b Médias seguidas de mesma letra não diferem estatisticamente pelo teste Tukey $(P>0,05)$.

$a, b$ Means followed by the same letter within row do not differ statistically by Tukey test $(P>0.05)$.

Os valores de conversão alimentar da MS, PB, FDN e do NDT ( $\mathrm{kg}$ de alimento consumido/kg de ganho de peso) são descritos na Tabela 4. As conversões alimentares da MS, da PB, da fração fibrosa e do NDT não diferiram entre as dietas com óleo de soja e com grão de soja. Contudo, com estas duas dietas, a conversão foi melhor que para aquela sem lipídeo adicional. $\mathrm{O}$ aporte energético proporcionado pela dieta com óleo de soja pode justificar a melhor conversão observada neste tratamento, que resultou em maior ganho de peso. Os valores de conversão da MS observados nesta pesquisa são comparáveis aos encontrados por Villares et al. (1981), Velloso et al. (1994), Jorge et al. (1997), Rodrigues et al. (2001) e Franzolin et al. (2001), em estudos sobre desempenho de bubalinos em confinamento.

Nas Tabelas 5 e 6 constam os custos com alimentação por animal por dia e a avaliação econômica do confinamento, respectivamente.

A dieta contendo grão de soja apresentou o maior custo com alimentação/animal/dia ( $\mathrm{R} \$ 2,77)$, o que fez com que representasse, na avaliação econômica, a dieta menos desejável. Esse resultado pode ser atribuído ao fato de o grão de soja ter, à época, alto valor comercial em relação a outros produtos utilizados na alimentação animal.

Os resultados indicaram que, apesar de elevar o custo com alimentação em relação à dieta sem lipídeo adicional, a dieta com óleo de soja é economicamente viável, pois os animais apresentaram ganho médio diário e rendimento de carcaça superiores aos obtidos com os demais tratamentos, provavelmente em decorrência do maior aporte de lipídios.

Pressupôs-se que, em regime de pastagem, os novilhos bubalinos ganhariam $450 \mathrm{~g} / \mathrm{dia}$, o que os levaria a despenderem de 184 a 239 dias a mais para ganharem os mesmos pesos obtidos pelos novilhos em confinamento. Nesta pesquisa, a liberação das pastagens em virtude do confinamento se converteu na maior fonte de recursos financeiros para os produtores que optarem pelo confinamento, 
Tabela 5 - Custos com alimentação/animal/dia no segundo semestre de $2003^{1}$

Table 5 - Daily feeding costs per animal in the second semester of 2003

\begin{tabular}{|c|c|c|c|c|}
\hline \multirow[t]{2}{*}{$\begin{array}{l}\text { Alimento } \\
\text { Feed }\end{array}$} & \multirow[t]{2}{*}{$\begin{array}{c}\text { Custo }(\mathrm{R} \$ / \mathrm{kg}) \\
\text { Cost }(R \$ / \mathrm{kg})\end{array}$} & \multirow[t]{2}{*}{$\begin{array}{l}\text { Sem lipídeo adicional } \\
\text { No added fat }\end{array}$} & $\begin{array}{l}\text { Grão de soja integral } \\
\text { Whole soybean }\end{array}$ & \multirow[t]{2}{*}{$\begin{array}{c}\text { Óleo de soja } \\
\text { Soybean oil }\end{array}$} \\
\hline & & & $\begin{array}{l}\text { sumo ( } \mathrm{kg} \text { de matéria natural) } \\
\text { Intake ( } \mathrm{kg} \text { of fresh matter) }\end{array}$ & \\
\hline Silagem de milho (Corn silage) & 0,04 & 21,69 & 20,82 & 20,00 \\
\hline Milho grão moído (Ground corn grain) & 0,27 & 4,00 & 2,93 & 3,37 \\
\hline Farelo de soja (Soybean meal) & 0,70 & 0,42 & 0,00 & 0,45 \\
\hline Uréia (Urea) & 0,72 & 0,11 & 0,00 & 0,10 \\
\hline Grão de soja (Whole soybean) & 0,75 & 0,00 & 1,53 & 0,00 \\
\hline Óleo de soja (Soybean oil) & 1,25 & 0,00 & 0,00 & 0,24 \\
\hline Custo/animal/dia (R\$) (Daily cost) & & 2,32 & 2,77 & 2,40 \\
\hline
\end{tabular}

${ }^{1}$ Valor do dólar à época: $\mathrm{R} \$ 2,90$.

${ }^{1}$ Dollar value at that time: $R \$ 2.90$.

Tabela 6 - Avaliação econômica do confinamento de bubalinos alimentados com diferentes fontes de lipídeos na dieta (no segundo semestre de 2003) ${ }^{1}$

Table 6 - Economical evaluation of feedlot water buffaloes fed diets with different fat sources (second semester of 2003)

\begin{tabular}{|c|c|c|c|}
\hline Item & $\begin{array}{l}\text { Sem lipídeo adicional } \\
\text { No added fat }\end{array}$ & $\begin{array}{c}\text { Grão de soja integral } \\
\text { Whole soybean }\end{array}$ & $\begin{array}{l}\text { Óleo de soja } \\
\text { Soybean oil }\end{array}$ \\
\hline Custo com alimentação/animal/dia (R\$) & 2,32 & 2,77 & 2,40 \\
\hline $\begin{array}{l}\text { Feeding cost/animal/day }(R \$) \\
\text { Dias de confinamento } \\
\text { Days in feedlot }\end{array}$ & 84,00 & 84,00 & 84,00 \\
\hline $\begin{array}{l}\text { Custo com alimentação total/animal ( } \mathrm{R} \$ \text { em } 84 \text { dias) } \\
\text { Total feeding cost/animal ( } R \$ \text { in } 84 \text { days) }\end{array}$ & 194,95 & 232,80 & 201,34 \\
\hline $\begin{array}{l}\text { Ganho médio diário (kg/animal) } \\
\text { Average daily gain }\end{array}$ & 0,99 & 1,09 & 1,28 \\
\hline $\begin{array}{l}\text { Ganho médio total (kg/animal) } \\
\text { Total daily gain }\end{array}$ & 83,16 & 91,56 & 107,52 \\
\hline $\begin{array}{l}\text { Rendimento de carcaça }(\%) \\
\text { Carcass yield }\end{array}$ & 51,69 & 48,93 & 52,24 \\
\hline $\begin{array}{l}\text { Ganho de peso em carcaça (@) } \\
\text { Carcassweight gain }\end{array}$ & 3,10 & 3,42 & 4,01 \\
\hline $\begin{array}{l}\text { Preçorecebido/@(R\$) } \\
\text { Grossincome/@ }\end{array}$ & 56,00 & 56,00 & 56,00 \\
\hline $\begin{array}{l}\text { Preço recebido/ganho total }(\mathrm{R} \$) \\
\text { Gross income/total gain }\end{array}$ & 173,86 & 191,42 & 224,79 \\
\hline $\begin{array}{l}\text { Ganho pressuposto em pastagem (kg/animal/dia) } \\
\text { Predicted gain on grazing (kg/animal/day) }\end{array}$ & 0,45 & 0,45 & 0,45 \\
\hline $\begin{array}{l}\text { Tempo necessário para ganhar o mesmo peso ganho } \\
\text { em confinamento, em pastagem (dias) } \\
\text { Time spent on pasture to gain the same feedlot weight (days) }\end{array}$ & 184,80 & 203,47 & 238,93 \\
\hline $\begin{array}{l}\text { Tempo necessário para ganhar em pastagem o mesmo } \\
\text { peso ganho em confinamento (meses) }\end{array}$ & 6,16 & 6,78 & 7,96 \\
\hline Time spent on pasture to gain the same feedlot weight (months) & & & \\
\hline $\begin{array}{l}\text { Aluguel de pastagem (R\$/animal/mês) } \\
\text { Pasture rent (R\$/animal/month) }\end{array}$ & 10,00 & 10,00 & 10,00 \\
\hline $\begin{array}{l}\text { Lucratividade com aluguel de pastagens liberadas } \\
\text { em virtude do confinamento }(\mathrm{R} \$) \\
\text { Profitability with pasture rent }\end{array}$ & 61,60 & 67,82 & 79,64 \\
\hline $\begin{array}{l}\text { Lucratividade com venda da carne (R } \$ \text { /animal) } \\
\text { Profitability with meat sales }\end{array}$ & $-21,09$ & $-41,38$ & 23,45 \\
\hline $\begin{array}{l}\text { Lucratividade total: venda da carne }+ \text { aluguel (R\$animal) } \\
\text { Profitability with meat sales }+ \text { pasture rent }\end{array}$ & 40,51 & 26,45 & 103,09 \\
\hline
\end{tabular}

1 Valor do dólar à época: $\mathrm{R} \$ 2,90$.

${ }^{1}$ Dollar value at that time: $R \$ 2.90$. 
principalmente com o emprego da dieta com óleo de soja, a qual proporcionou maior ganho de peso e abate em idade mais precoce. O confinamento geralmente proporciona, ainda, melhor qualidade da carne (Ramos, 2003), o que deve ser considerado, visto que alguns produtores têm obtido preço até $25 \%$ superior, atribuído à melhor qualidade da carne. Contudo, ao se recomendar uma das dietas estudadas nesta pesquisa, deve-se considerar que, segundo Gagleazzil et al. (2003), geralmente ocorre um deságio de aproximadamente $5 \%$ no preço da carne bubalina, justificado pelo menor rendimento da carcaça e pelo menor aproveitamento do couro. Além disso, a carne de búfalos ainda não é conhecida no mercado consumidor e não tem cadeia produtiva organizada.

Neste experimento, a dieta com óleo de soja foi a mais economicamente eficiente, pois, apesar de nenhuma das dietas possibilitarem lucratividade direta (por quilograma de carne produzido), outros benefícios indiretos ao confinamento foram observados, entre eles, os descritos por Pascoal et al. (1999): aceleração do giro de capital, redução da carga animal das pastagens durante a seca, possibilidade de programação da data de comercialização dos animais e abate de animais em idade mais precoce.

\section{Conclusões}

O uso do óleo de soja na dieta de bubalinos em confinamento se mostrou adequado, tendo em vista os melhores resultados de ganho de peso, conversão alimentar e resposta econômica, em comparação à ausência de lipídeo adicional e à inclusão de grão de soja na dieta.

\section{Literatura Citada}

ASSOCIATION OF OFFICIAL ANALYTICAL CHEMISTS - AOAC Official methods of analysis. 12.ed. Washington, D.C.: 1990. $1094 \mathrm{p}$.

ANUALPEC 2004. Anuário da pecuária brasileira. São Paulo: FNP Consultoria \& Comércio, 2004. 359p.

CHOUINARD, P.Y.; CORNEAU, L.; BUTLER, L.W.R. et al. Effect of dietary lipid source on conjugated linoleic acid concentrations in milk fat. Journal of Dairy Science, v.84, p.680-690, 2001.

DOREAU, M.; FERLAY, A. Effect of dietary lipids on nitrogen metabolism in the rumen: a review. Livestock Production Science, v.43, p.97-110, 1995.

FATURI, C.; RESTLE. J.; PASCOAL, L.L. et al. Avaliação econômica de dietas com diferentes níveis de substituição do grão de sorgo por grão de aveia preta para terminação de novilhos em confinamento. Ciência Rural, v.33, p.937-942, 2003.

FRANZOLIN, R.; SILVA, J.R.; CAMPOS, D. et al. Níveis de energia na dieta para bubalinos em crescimento alimentados em confinamento. 1. Desempenho e bioquímica de nutrientes sangüíneos. Revista Brasileira de Zootecnia, v.30, p.18721879, 2001.

GAGLEAZZIL, U.A.; GARCIAL, F.T.; BLISKA, F.M.M. et al Produção e consumo de carne bubalina no Brasil. Revista da Carne, n.314, p.32-39, 2003.

GOERING, H.K.; Van SOEST, J.P. Forage fiber analysis (apparatus reagents, procedure, and some applications). Washington, D.C.: Agriculture Handbook, ARS, USDA, 1970. p.379.

HOBSON, P.N.; STEWART, C.S. The rumen microbial ecosystem. London: Blackie Academic and Professional, 1997. 340p.

JENKINS, T.C.; McGUIRE, M.A. Major advances in nutrition: impact on milk composition. Journal of Dairy Science, v.89, p.1302-1310, 2006 .

JORGE, A.M. Produção de carne bubalina. In: OLIVEIRA, R.L. (Ed.) A zootecnia e o agronegócio. 1.ed. Brasília: Gráfica e Editora Inconfidência, 2004. v.1, p.617-634.

JORGE, A.M.; FONTES, C.A.A.; FREITAS, J.A. et al. Ganho de peso e de carcaça, consumo e conversão alimentar de bovinos e bubalinos, abatidos em dois estágios de maturidade. Revista Brasileira de Zootecnia, v.26, p.806-812, 1997.

NATIONAL RESEARCH COUNCIL - NRC. Nutrient requirements of dairy cattle. 7.rev.ed. Washington, D.C.: National Academic Press, 2001. 242p.

PACHECO, P.S.; RESTLE, J.; VAZ, F.N. et al. Avaliação econômica da terminação em confinamento de novilhos jovens e superjovens de diferentes grupos genéticos. Revista Brasileira de Zootecnia, v.35, p.309-320, 2006.

PALMQUIST, D.L. The role of dietary fats in efficiency of ruminants. Conference: regulating lipids metabolism to increase productive efficiency. Journal of Nutrition, v.124, p.1377$1382,1994$.

PASCOAL, L.L.; VAZ, R.Z.; ROSO, C. Confinamento versus pastagem na terminação de bovinos. In: RESTLE, J. (Ed.). Confinamento, pastagens e suplementação para produção de bovinos de corte. Santa Maria: Universidade Federal de Santa Maria, 1999. p.178-190.

RAMOS, A.A. Contribuição ao estudo dos bubalinos: 1972 2001/Palestras. Botucatu: [s.n.], 2003. p.202.

RODRIGUES, V.C.; ANDRADE, I.F.; GONÇALVES, T.M. et al Desempenho comparativo de bubalinos e bovinos em confinamento. Ciência e Agrotecnologia, v.25, p.396-407, 2001.

TAMMINGA, S.; DOREAU, M. Lipids and rumen digestion. In: JOUANY, J.P. (Ed.). Rumen microbial metabolism and ruminant digestion. Paris: Institut National de la Recherche Agronomique, 1991. p.151-164,

Van SOEST, P.J.; ROBERTSON, J.B.; LEWIS, B.A. Methods for dietary fiber, and nonstarch polysaccharides in relation to animal nutrition. Journal of Dairy Science, v.74 p.3583-3597, 1991.

VELLOSO, L.; SCHALCH, E.; FRANZOLIN NETO, R. et al. Desempenho comparativo de zebuínos Nelore e bubalinos Mediterrâneo em regime de confinamento. Revista Brasileira de Zootecnia, v.23, p.236-241, 1994.

VILlareS, J.B.; MOURA, J.C.; RAMOS, A.A. et al. Prova de ganho de peso de búfalos de raças Jafarabadi e Murrah. Os búfalos. Piracicaba: Fundação de Estudos Agrários Luiz de Queiroz, 1981. 185p. 